\title{
Islet cell antibody heterogeneity among Type 1 (insulin-dependent) diabetic patients
}

\author{
J. Timsit, S. Caillat-Zucman, H. Blondel, P. Chédin, J. F. Bach and C. Boitard \\ Department of Clinical Immunology, INSERM U 25, Hôpital Necker, Paris, France
}

\begin{abstract}
Summary. Conventional detection of islet cell antibodies is based on indirect immunofluorescence performed on frozen human pancreas sections. The number and nature of epitopes recognized by antibodies detected by such techniques are unknown. To determine the existence of heterogeneous fluorescence patterns of islet cell antibodies on pancreatic sections, we selected two sera showing a distinctive granular fluorescence. We then tested random sera from patients with Type 1 (insulin-dependent) diabetes mellitus for their ability to block ultimate binding of fluorescein isothiocyanatelabelled immunoglobulins purified from these two sera with a characteristic granular pattern. Among 102 subjects with
\end{abstract}

recent-onset Type 1 diabetes, 79 had detectable anti-islet cell antibodies; 21 showed complete blockade of the binding to islets of granular fluorescein isothiocyanate-labelled immunoglobulins. The majority of these 21 patients were women carrying a DR3 non-DR4 DQB1*0201 allele, with underrepresentation of DRB1*0402 and 0405. Discrimination between islet cell antigenic specificities may help in identifying islet cell autoantibodies in autoimmune Type 1 diabetes.

Key words: Islet cell antibodies, antigens, immunofluorescence, HLA.
Islet cell antibodies (ICA) are detected in the serum of most recent-onset Type 1 (insulin-dependent) diabetic patients and in first-degree relatives of diabetic patients months or years before the onset of clinical signs. In subjects at risk, the predictive value of ICA for the occurrence of Type 1 diabetes depends on ICA titres [1]. Conventional techniques for detecting ICA are mostly based on indirect immunofluorescence performed on frozen human pancreas sections [2]. The precision of ICA detection has improved with the advent of international standardization programmes which have established an international unit [3], but a significant level of uncertainty persists partly due to the multiplicity and the loose definition of the target antigens. Thus, these antigens need to be more clearly defined.

ICA generally recognize antigens that have been described as including glycolipids expressed on all cell types present in the islet of Langerhans, although some sera selectively recognize Beta cells, detected by double labelling with anti-insulin antibodies (restricted ICA) [4]. Other autoantibodies have been detected in diabetic and pre-diabetic subjects, including antibodies to insulin (ELISA and immunoprecipitation of iodine-labelled insulin) and to a $64 \mathrm{kDa}$ antigen (immunoprecipitation of islet antigen extracts) [5]. Among the anti-64 kDA anti- bodies, those directed against glutamic acid decarboxylase (GAD) have been further characterized [6]. The question has been raised whether total or restricted ICA include some of the above-mentioned antibodies.

In this report we have approached the heterogeneity of ICA by studying the morphological pattern of immunofluorescence using a conventional ICA assay. We have thus distinguished granular and homogeneous fluorescence distribution patterns. Immunoglobulins from two sera which gave a reproducible granular pattern were purified and fluorescein isothiocyanate (FITC)-labelled, and subsequently used in a blocking assay in order to further characterize ICA subtypes in a large population of adult patients with recent-onset Type 1 diabetes.

\section{Patients and methods}

\section{Patients}

A group of 102 adults with recent-onset Type 1 diabetes (symptoms of less than 4 months duration) comprising 70 males and 32 females aged $25.2 \pm 0.82$ years (mean $\pm \mathrm{SEM}$ ), were included in this study. Serum samples were taken at first examination of the patient and stored at $-80^{\circ} \mathrm{C}$. 


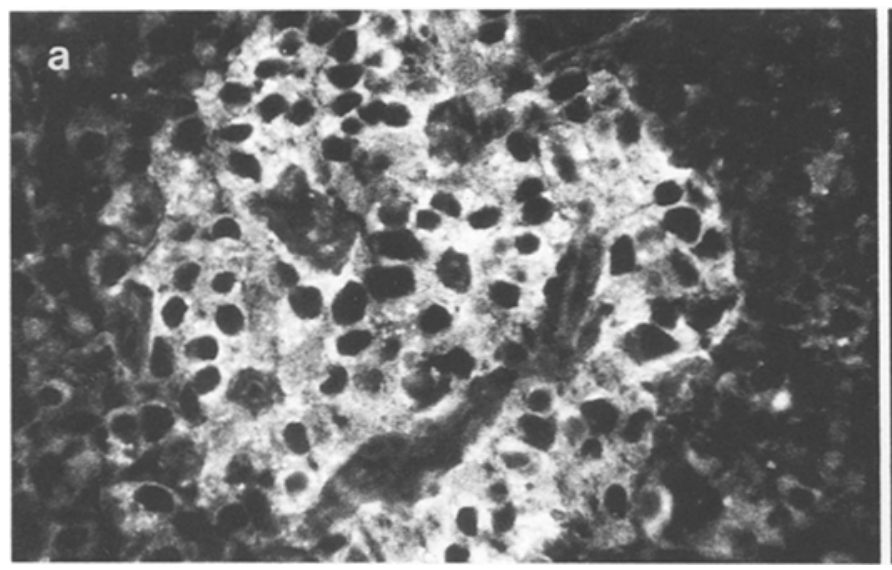

Fig. 1a,b. Islet cell antibody fluorescence patterns on frozen human pancreas sections. Sera from patients with Type 1 (insulin-dependent) diabetes were tested with a conventional islet cell antibody

\section{ICA determination}

Total ICA and complement-fixing ICA were assayed as described by Bottazzo et al. [2]. Sera were also tested after prolonged $(18 \mathrm{~h})$ incubation in the presence of aprotinin (S-ICA) [7]. The precision and accuracy of our ICA determination were evaluated within international workshops (assay $\$ 1$ in the 3rd ICA Workshop) [3]. The detection threshold of our technique was below 5 Juvenile Diabetes Foundation (JDF) units. The specificity of ICA detection for Type 1 diabetes was $99.7 \%$ in a random population of 5,584 men aged 18 20 years. Results are expressed as the $\log ^{2}$ of ICA JDF units determined in the conventional assay using the international standard [3]. Double immunostaining techniques were performed as described elsewhere [8], using anti-insulin (OXI 002), anti-somatostatin (SOM 018) and anti-glucagon (GLU 001) monoclonal antibodies (Novo, Boulogne, Billancourt, France)

\section{Blocking assay}

Frozen human pancreas sections were first incubated with the undiluted test sera for $25 \mathrm{~min}$ at $22^{\circ} \mathrm{C}$, then washed with phosphate buffered saline and incubated for $30 \mathrm{~min}$ with FITC-labelled immunoglobulins (gICA-FITC A and B) purified, by protein A-Sepharose column chromatography, from two recent-onset diabetic patients and giving a typical granular pattern in the conventional assay. The gICAFITC A and B reagents thus replaced the FITC-labelled goat antihuman Ig antiserum used in the conventional ICA assay. A and B FITC conjugates were positive up to $1: 32$ and $1: 128$ dilutions, respectively. They were used in the blocking assay at 1:16 and 1:50 dilutions, respectively, corresponding to calibration at 10 JDF units with reference to the international standard provided in the 3rd ICA Workshop [3]. Sera were tested with the conventional ICA and the blocking assay without prior knowledge of sample identity and were referred to as "blocking" or "non-blocking" in the latter assay. The conjugation of purified immunoglobulins obtained from sera giving a homogeneous pattern did not allow for direct staining on pancreas sections. This precluded the study of sera from diabetic patients in a blocking assay similar to that reported with gICA-FITC.

\section{HLA typing}

HLA DRB, DQA and DQB alleles were determined by means of oligotyping following DNA amplification and using allele-specific probes as described elsewhere [9].

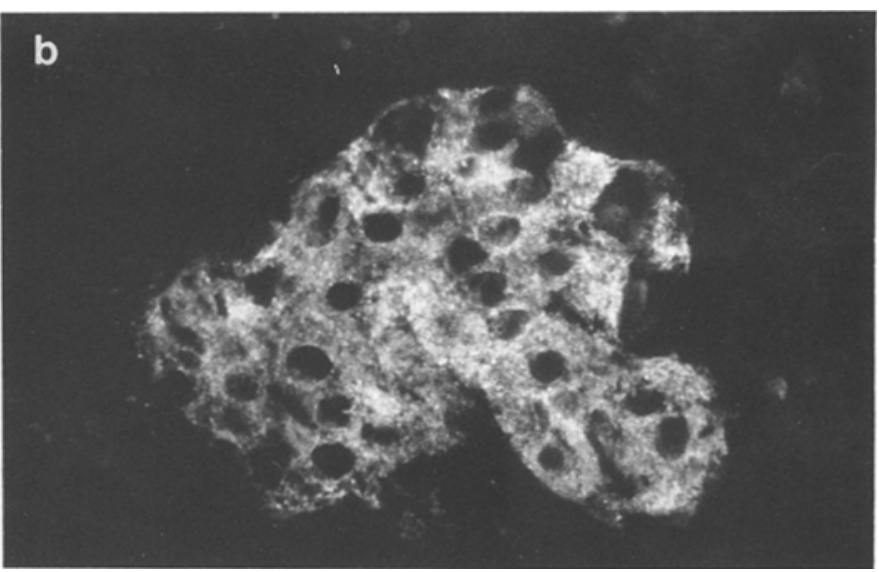

assay. The majority showed a homogeneous distribution of fluorescence within the islet cell cytoplasm (a), while a granular pattern was observed with the others $(\mathbf{b})$. Original magnification $\times 127$

\section{Statistical analysis}

Student's $t$-test and the chi square method were used.

\section{Results}

\section{ICA fluorescence patterns}

Two ICA fluorescence patterns were identified: the first was a diffuse, homogeneous pattern (Fig. 1 a) and the second a granular pattern with irregular fluorescence within the islet cell cytoplasm, scattered in discrete spots (Fig. 1b). These distinct patterns were observed using three different pancreata. Both patterns were found in insulin- and non-insulin-secreting islet cells, as shown by double staining using anti-insulin, anti-somatostatin, and anti-glucagon monoclonal antibodies, but some glucagonsecreting islet cells were clearly not stained by sera giving the granular pattern. The fluorescence patterns were not simply related to the ICA titre, since serial dilution of the sera did not modify the fluorescence distribution. However, the discrimination between the two fluorescence patterns was often difficult by direct examination of pancreas sections, particularly with sera containing low titre ICA (data not shown). This prompted us to use a blocking assay in order to further characterize the tested sera.

\section{Blocking assay}

The two reference sera were checked for their ability to provide the same granular fluorescence pattern after FITC conjugation as before conjugation. Each unlabelled serum (A and B) completely inhibited gICA-FITC A and $B$ staining and showed cross-blocking in the blocking assay. Among sera from 102 patients with recent-onset Type 1 diabetes (Table 1), $60(58.8 \%$ ) were ICA-positive in the conventional assay, $37(36.3 \%)$ were complementfixing and $79(77.4 \%)$ were positive after prolonged incubation in the presence of aprotinin (S-ICA).

None of the S-ICA-negative sera inhibited the binding of gICA-FITC. Among the S-ICA positive sera, 21 $(26.6 \%)$ were completely "blocking", $42(53.2 \%)$ were 
Table 1. Main characteristics of islet cell antibody (ICA)-positive Type 1 (insulin-dependent) diabetic patients

\begin{tabular}{llll}
\hline Patient group & $\begin{array}{l}\text { Complete } \\
\text { blocking }\end{array}$ & $\begin{array}{l}\text { Partial } \\
\text { blocking }\end{array}$ & $\begin{array}{l}\text { No } \\
\text { blocking }\end{array}$ \\
\hline$n$ & 21 & 16 & 42 \\
ICA + & 19 & 12 & 30 \\
CF-ICA + & $15^{\mathrm{a}}$ & 4 & 18 \\
S-ICA +, ICA- & 2 & 4 & 12 \\
ICA titre ( $\log ^{2}$ JDF units) & $5.89 \pm 2.89^{\mathrm{a}}$ & $4.19 \pm 1.99$ & $4.49 \pm 2.53$ \\
Female/male & $11 / 10^{\mathrm{a}}$ & $5 / 11$ & $10 / 32$ \\
DR3/non DR4 & $10 / 21^{\mathrm{a}}$ & $6 / 16$ & $9 / 42$ \\
DR4/non DR3 & $7 / 21$ & $6 / 16$ & $16 / 42$ \\
DR3/DR4 & $3 / 21$ & $3 / 16$ & $13 / 42$ \\
DQB1*0201/non *0302 & $12 / 21$ & $7 / 16$ & $13 / 41$ \\
DRB1*0401 (DW 4) & $8 / 10^{\mathrm{a}}$ & $5 / 8$ & $10 / 28$ \\
DRB1*0402 (DW 10) & $0 / 10$ & $1 / 8$ & $7 / 28$ \\
DRB1*0405 (DW 15) & $1 / 10$ & $2 / 8$ & $9 / 28$ \\
\hline
\end{tabular}

a $p<0.05$ vs patients with non-blocking sera. The three groups of patients were defined in terms of the ability of their serum to block binding to islet cells of FITC-labelled immunoglobulins that gave a granular fluorescence pattern in a conventional ICA assay (see Methods). No serum in which ICA were not detected, even in a sensitized assay after prolonged incubation, provided a significant blockade. CF, Complement-fixing

"non-blocking" and 16 (20.2\%) showed partial blocking. All the sera had identical blocking or non-blocking effects on gICA-FITC A and B staining. There was no absolute correlation between the fluorescence pattern (homogeneous vs granular) and the blocking ability. A number of sera with homogeneous pattern were able to provide a complete blocking effect.

\section{Clinical correlations}

We compared the two groups of patients delineated by a clear-cut pattern in the blocking assay (i.e. blocking and non-blocking of gICA-FITC binding). Patients whose sera showed partial blocking were not included in these comparisons since the significance of this pattern may not be uniquivocal.

Blocking sera contained higher ICA titres and were more often complement-fixing $(p<0.05$, Table 1$)$. However, when considered individually, two sera which were only positive after prolonged incubation (but not in the conventional assay) contained "blocking" antibodies (Table 1)

There were twice as many women in the group with blocking antibodies than in that with non-blocking antibodies $(p<0.05)$.

The frequency of DR3/non-DR4 alleles and DQB $1 * 0201$ was higher $(p<0.05)$ in the patients with blocking ICAs than in those with non-blocking ICAs (Table 1). HLA-DR4 subtyping showed that the patients with blocking ICAs were more frequently DRB $1 * 0401$ than those with non-blocking ICAs $(p<0.05)$. However, since the $p$ value was not corrected for the number of alleles analysed it will be necessary to confirm these results in a larger population of patients.

\section{Discussion}

Immunofluorescence assays for ICA detection are currently used to detect antibodies against islet cells in patients with recent-onset Type 1 diabetes or in individuals at risk. Antibodies to insulin, proinsulin, GAD, carboxypeptidase $\mathrm{H}$, and antigens with ubiquitous tissue distribution have since been identified $[4,5]$. One may thus assume that ICA include antibodies with different antigenic specificities as detected by conventional indirect immunofluorescence techniques. This heterogeneity has been previously suggested in pre-diabetic subjects and patients with autoimmune polyendocrinopathies $[4,10]$.

We have found that at least two different patterns of reactivity could be observed on tissue sections among ICApositive sera, independently of the dilution tested. The majority of the sera showed a homogeneous pattern and some a clear granular pattern. A blocking assay was used for accurate discrimination of the two ICA subtypes. The two sera used for the preparation of gICA-FITC A and B contained a similar array of antibodies, since they were completely cross-reactive in the blocking assay and all the sera from diabetic patients provided consistent results when tested with the two gICA-FITC reagents. Among the diabetic patients a subgroup could be identified by the ability of their serum to block the binding of the two reference sera with a typical granular fluorescence pattern. It is important to note that some sera giving a homogeneous pattern were blocking, suggesting that in addition to other antibodies they may contain antibodies binding to the epitopes recognized by the exclusively granular antibodies. Sera that only partially inhibited the binding of gICAFITC A and B may contain antibodies with low avidity or alternatively low antibody titres. However, some sera with low ICA titres (i.e. positive only after prolonged incubation) contained blocking antibodies. In the hypothesis that several antigenic specificities are encompassed by "granular" antigens, one may also assume that sera showing partial blockade may only recognize part of these "granular" antigenic specificities.

Sera containing antibodies restricted to Beta cells ("selective pattern") [4] and staining human and rat but not mouse pancreas [10] have been distinguished as different from sera containing antibodies that bind to all islet cells. In one study, sera containing such restricted ICA gave a granular immunofluorescence pattern on frozen human pancreas sections [4]. Whether restricted ICA and the granular antibodies detected in our blocking assay represent similar or overlapping entities remains to be determined. It is noteworthy that some sera containing "blocking" antibodies did not bind to all glucagon-secreting islet cells, but somatostatin-secreting islet cells were clearly stained. Beta-cell restricted ICA have been reported in patients with autoimmune polyendocrinopathy and in relatives of patients with Type 1 diabetes who do not develop diabetes $[4,10]$. In our study, sera with granular antibodies were observed in $26.6 \%$ of recent-onset Type 1 diabetic patients (predominantly women and DR3 patients) but we found no association with polyendocrine autoimmunity. It should be noticed that all the patients in our study were adults who represent a restricted group regarding age at 
onset. Preliminary results indicate that "blocking" antibodies are also present in children with recent-onset Type 1 diabetes. We are now evaluating subjects at risk of disease (relatives of diabetic patients) in order to determine the predictive value of the two ICA patterns reported in this study. The blocking assay described here identifies the existence of a subtype of ICA; existing exclusively or prevalently in some sera (the granular fluorescence pattern), coexisting with other autoantibodies in some cases (the homogeneous pattern) and otherwise undetectable. The identification of different ICA specificities and their correlation with other autoantibodies such as anti-insulin, anti-64kDa and anti-GAD will help to clarify the autoantigen-antibody networks seen in Type 1 diabetes.

Acknowledgements. We thank M. F. Richard for technical assistance and $D$. Young for critical review of the manuscript.

\section{References}

1. Bonifacio E, Bingley PJ, Shattock M et al. (1990) Quantification of islet-cell antibodies and prediction of insulin-dependent diabetes. Lancet 335: 147-149

2. Bottazzo GF, Florin-Christensen A, Doniach D (1974) Islet cell antibodies in diabetes mellitus with autoimmune polyendocrine deficiency. Lancet II: 1279-1283

3. Bonifacio E, Boitard C, Gleichmann H et al. (1990) Assessment of precision, concordance, specificity, and sensitivity of islet cell antibody measurement in 41 assays. Diabetologia 33: 731-736
4. Bottazzo GF, Genovese S, Bosi E, Dean BM, Christie MR, Bonifacio $E$ (1991) Novel consideration on the antibody/autoantigen system in type 1 (insulin-dependent) diabetes mellitus. Ann Med 23: 453-461

5. Riley WJ, Maclaren NK (1991) Islet cell autoantibodies. In: Samols E (ed) The endocrine pancreas. Raven Press, New York, pp 409-422

6. Baekkeskov S, Aanstoot HJ, Christgau S et al. (1990) Identification of the $64 \mathrm{~K}$ autoantigen in insulin-dependent diabetes as the GABA-synthesizing enzyme glutamic acid decarboxylase. Nature 347: 151-156

7. Pilcher C, Elliott RB (1984) Improved sensitivity of islet cell cytoplasmic antibody assay in diabetics. Lancet I: 1352

8. Bottazzo GF, Dean BM, McNally JM, MacKay EH, Swift PGF, Gamble DR (1985) In situ characterization of autoimmune phenomena and expression of HLA molecules in the pancreas in diabetic insulitis. N Engl J Med 313: 353-360

9. Tiercy JM, Goumaz C, Mach B, Jeannet M (1991) Application of DR oligotyping to 110 kidney transplant patients with doubtful serological typing. Transplantation 51:110-114

10. Gianani R, Pugliese A, Bonner-Weir S et al. (1992) Prognostically significant heterogeneity of cytoplasmic islet cell antibodies in relatives of patients with type 1 diabetes. Diabetes 41:347-353

Received: 23 March 1992

and in revised form: 16 April 1992

Prof. C. Boitard

INSERM U 25

Hôpital Necker

161 rue de Sèvres

F-75743 Paris Cedex 15

France 